\title{
Primary Spinal Cord Melanoma of Intradural Extramedullary Origin
}

\author{
Ashish Sharma ${ }^{1}$ Virendra Deo Sinha ${ }^{1}$ \\ ${ }^{1}$ Department of Neurosurgery, Sawai Man Singh Medical College, \\ Jaipur, Rajasthan, India
}

J Neurosci Rural Pract 2019;10:522-525

\begin{abstract}
Address for correspondence Virendra Deo Sinha, MCh, Department of Neurosurgery, Sawai Man Singh Medical College, Jaipur 302004, Rajasthan, India (e-mail: sinhavd@yahoo.com).
\end{abstract}

\begin{abstract}
Keywords

- extramedullary

- primary malignant melanoma

- spinal cord

Primary malignant melanoma is the very rare entity of the central nervous system. It accounts only $1 \%$ of all melanoma cases. A 67 -year-old patient presented to us with lower back pain radiating to the left lower limb and progressive weakness of both lower limbs. She underwent magnetic resonance imaging scan of lumbosacral region that suggested T1 hyperintense, T2 hypointense, extramedullary altered signal intensity lesion at L1 and L2 vertebral level compressing terminal spinal cord, and cauda equine region. On histopathological examination, findings were suggestive of malignant melanoma that was confirmed with immunohistochemistry examination (positive for S-100, Melan-A, and HMB-45). Here, we are discussing an unusual case of primary malignant melanoma of conus and cauda equine region with comprehensive review regarding management and prognosis of the tumor.
\end{abstract}

\section{Introduction}

Primary malignant melanoma is very rare entity of the central nervous system (CNS). They account only $1 \%$ of all melanoma cases. ${ }^{1-3}$ CNS is the third most common site for melanoma metastasis. ${ }^{4}$ Extramedullary primary malignant melanoma of spinal cord is one of the rare lesions, and thus till date, only 27 cases are published in the literature. ${ }^{5}$

Very little literature is available on primary malignant melanomas of the spinal cord; therefore, the regarding exact incidence, treatment, and prognosis are still unclear. Here, we are reporting a case of primary spinal cord melanoma of intradural extramedullary origin in a 67-year-old female patient.

\section{Case Report}

A 67-year-old female patient admitted to the neurosurgery department with lower back pain radiating to the left lower limb and progressive weakness of both lower limbs. Weakness first appeared in the left lower limb and gradually progressed to right lower limb.

Neurological examination revealed power in the left lower limb of grade 3/5 and right lower limb of grade $4 / 5$ according to MRC (Medical Research Council) scale. Deep tendon reflexes were normal, and sphincter function was intact. Sensations in the lower limb of the patient were also intact. She underwent magnetic resonance imaging (MRI) scan of lumbosacral (LS) region (plane plus contrast) suggestive of T1 hyperintense, T2 hypointense, extramedullary altered signal intensity lesion at L1 and L2 vertebral level compressing terminal spinal cord, and cauda equine region. Findings confirmed a mass of heterogeneously contrast enhancing and extending to the left extraforaminal region through the L1 and L2 neural foramen ( $\bullet$ Fig. 1A, B).

Dermatological, ophthalmological examination was performed to rule out any suspected melanotic lesion. There was no melanotic lesion found, after which it was considered as primary melanoma of the spinal cord. The patient was planned for surgery and the L1 to L3 laminectomy performed. After dural opening, a brown to black pigmented, vascular, and soft to firm mass was noted against L1 and L2 vertebral bodies. Mass was densely adhered to the terminal cord (conus), L2 nerve root, and extended outside through left neural foramen. Complete excision could not be performed because of adherence of mass to the left L1 nerve root. Postoperatively, the patient was doing well. The symptoms, back pain, and radiation of pain subsided. The patient was discharged after 7 days of surgery. 

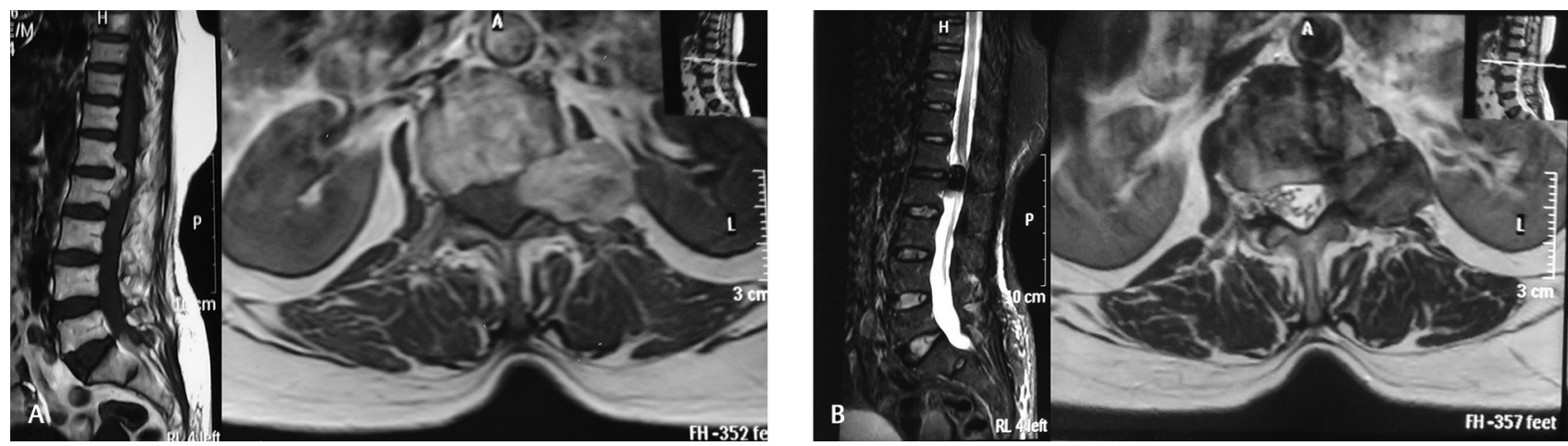

Fig. 1 (A) Magnetic resonance imaging lumbosacral spine-T1 sagittal and the axial view showing heterogeneous T1 hyperintense lesion at L1 and $\mathrm{L} 2$ vertebral level with the axial scan showing mass is exiting through left neural foramen compressing thecal sac to the right side. (B) T2 sagittal and the axial view showing mass is heterogeneous and hypointense.

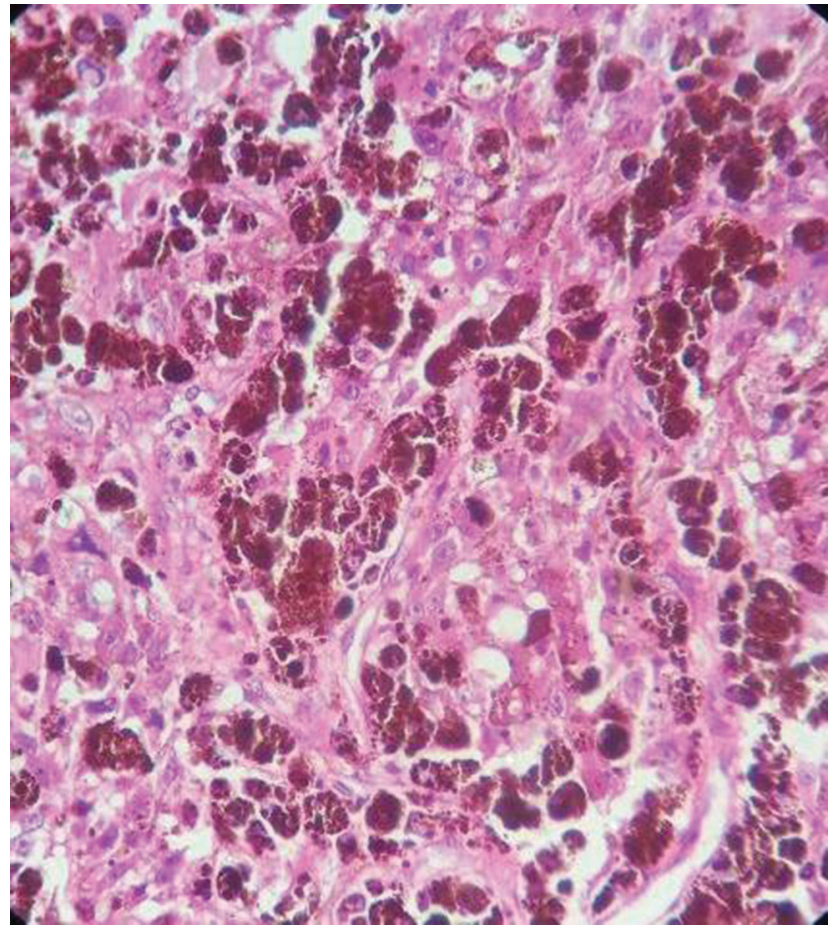

Fig. 2 Highly anaplastic tumor cells with the high degree of cytological atypia and occasional mitotic figures. Cells have large eosinophilic nucleoli, nuclear grooves, folds, and exhibit prominent melanin pigmentation, and it was suggestive of malignant melanoma.
Histopathological examination of the tumor tissue showed highly anaplastic tumor cells with the high degree of cytological atypia and occasional mitotic figures. Cells have large eosinophilic nucleoli, nuclear grooves, folds, and exhibit prominent melanin pigmentation, and it was suggestive of malignant melanoma ( - Fig. 2). This was further confirmed by immunohistochemistry of tissue showing marked positivity for S-100, Melan-A, and human melanoma black (HMB)-45.

After discharge, the patient did not come back for follow-up. After 9 months of surgery, the patient again presented with the same clinical features of back pain with radiation and motor weakness of lower limbs. MRI LS spine was performed that suggestive of recurrence of the tumor ( - Figs. $\mathbf{3 A}$ and $\mathbf{B})$. The multidisciplinary team decided for radiotherapy. Further on follow-up patient improved partially.

\section{Discussion}

Primary melanomas of CNS are quite rare with only approximately $1 \%$ occurrence worldwide. ${ }^{1}$ Previously reported case reports concluded that it can be derived from leptomeningeal melanoblasts accompanying the pial sheaths of vessels or from neural crest cells during embryogenesis., 4,6

As per the Hayward classification of primary melanoma is called primary when an absence of melanoma outside CNS, an absence of this lesion in other sites in CNS and diagnosis
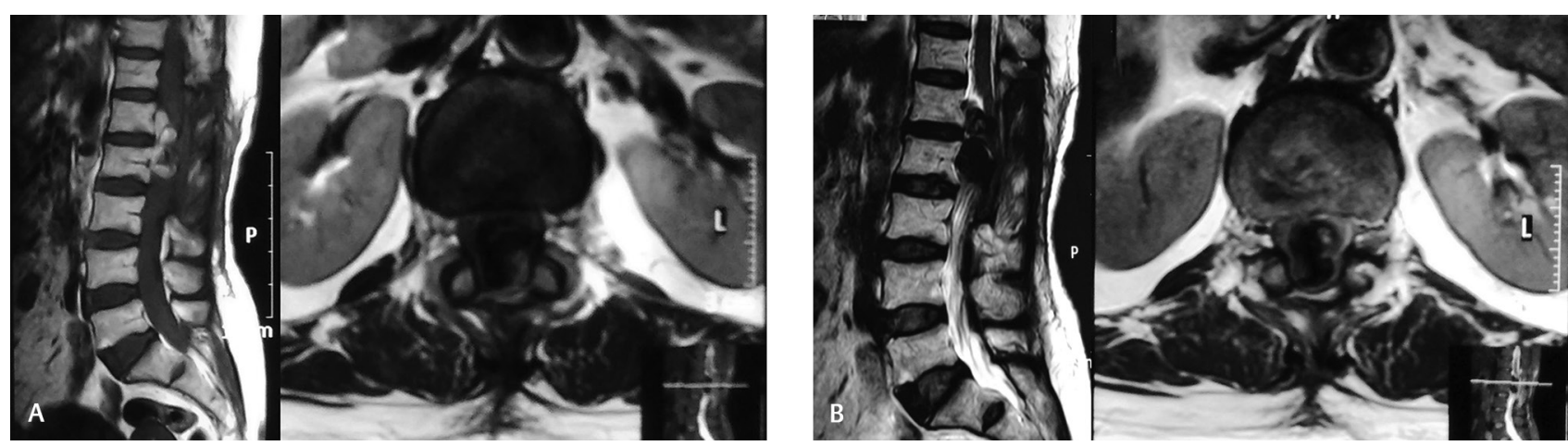

Fig. 3 (A) Magnetic resonance imaging T1-weighted image sagittal and axial view showing postoperative changes with the heterogeneous mass lesion at L1 and L2 levels suggestive of recurrent mass. (B) T2 sagittal and axial view. 
of melanoma confirmed by histopathology. ${ }^{7}$ Taking these criteria into consideration, our reported case was diagnosed as primary malignant melanoma of the spinal cord and more precisely of the conus medullaris. About $90 \%$ of melanomas of CNS were metastatic in origin. A thorough physical examination and radiological investigation are required to rule out melanoma in other organs outside of the spinal cord before a diagnosis of primary spinal melanoma can be established.

According to Liu et al, extramedullary primary spinal melanoma had slightly more preponderance in the cervical segments (39.3\%). ${ }^{5}$ While other authors, Farrokh et al and Cetinalp et al reported that malignant melanoma of intramedullary origin was more common in the thoracic region. ${ }^{1,8}$ Most of the patients were of middle-age group without any gender preference. The majority of tumors were solitary, and only a few cases were reported with multiple foci. ${ }^{9}$ As in our case, the extramedullary origin of melanoma, few tumors originated from the nerve roots or had spinal nerve root involvement, ${ }^{10,11}$ which led to the enlargement of the intervertebral foramen, and even spread outside the intervertebral foramen. ${ }^{12}$

The clinical presentation of these tumors was nonspecific and has similarity with other tumors, such as meningioma, neurofibroma, and degenerative disc disease. Clinically extramedullary malignant melanoma may be misdiagnosed as with other tumors, such as meningioma, ependymoma, schwannoma, or metastatic tumor. ${ }^{10-12}$

MRI is the modality of choice for establishing the diagnosis of spinal cord tumors. The varied signal intensity poses a challenge in the morphological and locational identification of these tumors. It has been observed that the imaging spectrum of these melanomas overlaps with other pigmented tumors such as melanocytic schwannoma and melanocytoma. ${ }^{4,8}$ The melanoma has different radiological characteristic signals (hyperintensity on T1-weighted [W] and hypointensity on T2-W) on MRI examination than other similar tumors, such as schwannoma or meningioma. ${ }^{13}$ On contrast MRI, these tumors showed a homogeneous enhancement of the lesion.

Although the treatment of extramedullary primary melanoma has not yet been standardized, the total surgical resection is the best modality of treatment. Radiotherapy (from 30-60 Gy) is effective for local control and is reserved for palliation or as an adjuvant to surgical resection. ${ }^{10-12,14,15}$ According to literature, it has not been established whether postoperative radiotherapy or chemotherapy (such as interferon, methotrexate, and temozolomide) has a significant role in overall survival time. ${ }^{10-12,14,15}$ Most of the systemic chemotherapies are refractory for these tumors. Owing to the relative rarity of these tumors, the role of immunotherapy is not yet documented in the literature; still immunotherapy is the current paradigm shift in the management of melanoma. Metastasis can also be there outside to CNS, especially in the brain, lung, bone, systemic metastasis, or the intraspinal metastasis. ${ }^{15-17}$

Because of limited follow-up data, the survival and prognosis are still unclear. It was observed in quite number of patients with primary CNS melanoma have a long-life expectancy, and some even-attain cure, while the mean survival for metastatic CNS melanoma is reported to be less than a year.

\section{Conclusion}

Primary spinal cord melanoma is very rare, and it is important to differentiate them from a metastatic cutaneous melanoma because these have relatively better prognosis. The preoperative diagnosis is primarily based on characteristic MRI findings (hyperintensity on T1-W and hypointensity on $\mathrm{T} 2-\mathrm{W}$ ). The primary treatment strategy for this tumor is complete excision, and additional postoperative radiotherapy or chemotherapy may be given if necessary. In this report, we believe that the clinician must be aware of the possibility of a primary malignant melanoma when the MRI images depict such a spinal cord tumor with paramagnetic properties.

\section{Funding \\ None. \\ Conflict of Interest \\ None declared.}

\section{References}

1 Farrokh D, Fransen P, Faverly D. MR findings of a primary intramedullary malignant melanoma: case report and literature review. AJNR Am J Neuroradiol 2001;22(10):1864-1866

2 Kounin GK, Romansky KV, Traykov LD, Shotekov PM, Stoilova DZ. Primary spinal melanoma with bilateral papilledema. Clin Neurol Neurosurg 2005;107(6):525-527

3 Jaiswal S, Vij M, Tungria A, Jaiswal AK, Srivastava AK, Behari S. Primary melanocytic tumors of the central nervous system: a neuroradiological and clinicopathological study of five cases and brief review of literature. Neurol India 2011;59(3):413-419

4 Kim MS, Yoon DH, Shin DA. Primary spinal cord melanoma. J Korean Neurosurg Soc 2010;48(2):157-161

5 Liu QY, Liu AM, Li HG, Guan YB. Primary spinal melanoma of extramedullary origin: a report of three cases and systematic review of the literature. Spinal Cord Ser Cases 2015;1:15003

6 Pappenheim E, Bhattacharji SK. Primary melanoma of the central nervous system. Clinical-pathological report of a case, with survey and discussion of the literature. Arch Neurol 1962;7:101-113

7 Hayward RD. Malignant melanoma and the central nervous system. A guide for classification based on the clinical findings. J Neurol Neurosurg Psychiatry 1976;39(6):526-530

8 Cetinalp NE, Yildirim AE, Divanlioglu D, Belen D. An uncommon intramedullary tumor: primary spinal cord melanoma. Asian Spine J 2014;8(4):512-515

9 Huang X, Pan X, Huang H, Zhan R. Multiple spinal cord melanoma: case report with emphasis on the difficult preoperative diagnosis. Turk Neurosurg 2013;23(4):534-538

10 Naing A, Messina JL, Vrionis FR, Daud AI. Uncommon manifestations of common malignancies: case 3. Malignant melanoma arising from a spinal nerve root. J Clin Oncol 2004;22(15):3194-3195

11 Kwon SC, Rhim SC, Lee DH, Roh SW, Kang SK. Primary malignant melanoma of the cervical spinal nerve root. Yonsei Med J 2004;45(2):345-348

12 Kanatas AN, Bullock MD, Pal D, Chakrabarty A, Chumas P. Intradural extramedullary primary malignant melanoma 
radiographically mimicking a neurofibroma. Br. J Neurosurg 2007;21(1):39-40

13 Smith AB, Rushing EJ, Smirniotopoulos JG. Pigmented lesions of the central nervous system: radiologic-pathologic correlation. Radiographics 2009;29(5):1503-1524

14 Lee $\mathrm{CH}$, Moon KY, Chung CK, et al. Primary intradural extramedullary melanoma of the cervical spinal cord: case report. Spine 2010;35(8):E303-E307

15 Cicuendez M, Paredes I, Munarriz PM, Hilario A, Cabello A, Lagares A. Primary melanoma of the cauda equina: case report and review of the literature. Neurocirugia (Astur) 2012;23(3):112-115
16 Yu J, Zhao DD, Chen S, Zhang JM, Xu J. Primary melanoma of the cervical spine with cerebral metastases: case report and review of the literature. J Int Med Res 2012;40(3):1207-1215

17 Katalinic D, Anic B, Stern-Padovan R, et al. Low back pain as the presenting sign in a patient with primary extradural melanoma of the thoracic spine-a metastatic disease 17 years after complete surgical resection. World J Surg Oncol 2011;9:150 